\title{
Weiter für Werte sensibilisieren
}

\section{Das bundesweite Projekt » Wertebildung in Familien « wird fortgesetzt}

\author{
Annegret Erbes, Charlotte Giese, Heribert Rollik
}

\begin{abstract}
Ziel eines Projekts des Deutschen Roten Kreuzes ist die Stärkung von Wertorientierungen in Familien angesichts einer von wachsender Heterogenität und Komplexität geprägten Gesellschaft. Im Vordergrund steht die Entwicklung von Strategien, die es Kindern ermöglichen, sich an Werte zu binden. Hierzu zählen vor allem Prozesse, die Eltern, Erzieherinnen und Erzieher darin unterstützen, ihre eigenen Werthaltungen zu reflektieren.
\end{abstract}

Die Auseinandersetzung mit Werten und mit Wertebildung hat eine lange Tradition - und wird trotzdem immer aktuell bleiben. Sich gemeinsam als Gesellschaft auf Werte zu beziehen, stärkt den sozialen Zusammenhalt. Werte sind jedoch auch ein wichtiges Thema für jeden einzelnen Menschen: In Krisen fragen wir nach dem, was uns im Leben wirklich wichtig ist, was uns Halt gibt und auch, was wir an die nachfolgende Generation weitergeben möchten.

Das Generalsekretariat des Deutschen Roten Kreuzes hat in Kooperation mit dem Bundesministerium für Familie, Senioren, Frauen und Jugend das Projekt »Wertebildung in Familien « initiiert. Bereits in der Pilotphase (2008 bis 2010) wurden bundesweit 15 Projektstandorte finanziell gefördert. Aufgrund der großartigen Arbeit an den Standorten und den guten Ergebnissen der Projektevaluation (Universität Erlangen-Nürnberg) wurde eine zweite Projektphase bis Ende 2011 vom Bundesministerium für Familie, Senioren, Frauen und Jugend bewilligt.

In Familien wird - im Zuge von Modernisierungsprozessen und Migration

Die Autoren sind Mitarbeiter des Deutschen Roten Kreuzes e. V. Internet http://www.drk.de heute mehr denn je - Wertevielfalt gelebt. Diese Vielfalt von Werten und Kulturen versteht moderne Familienbildung positiv und greift sie durch Angebots- und Methodenvielfalt auf, um für möglichst alle Familien - unabhängig von Herkunft, Bildungsstand und sozialer Lage - Angebote zu entwickeln und bereitzuhalten.

Wertebildung in Familien ist daher träger- und weltanschauungsübergreifend konzipiert und kann so der Vielfalt von Familienformen und Werten in Familien sowie der Vielfalt von Angeboten und Methoden moderner Familienbildung Rechnung tragen. Ebenso spiegeln die 15 Praxisstandorte die Vielfalt relevanter Handlungsfelder von Familienbildung wider. Das Deutsche Rote Kreuz (DRK) eignet sich besonders als Projektträger, da es der im Projekt angelegten Vielfalt mit seinen Grundsätzen Menschlichkeit, Unparteilichkeit, Neutralität, Unabhängigkeit, Freiwilligkeit, Einheit, Universalität sowie mit seiner fachlichen Kompetenz einen Rahmen geben kann. Im Folgenden soll das Vorhaben genauer vorgestellt werden.

\section{Projektphase 1: Mai 2008 bis Juni 2010}

Oberstes Ziel der ersten Phase des Projekts Wertebildung in Familien war es, Familien in ihren wertebildenden Prozessen

- vor dem Hintergrund ihrer eigenen Werte,

- anknüpfend an Bedürfnisse und Lebenswelten und

- mit verschiedensten Angeboten und Methoden

zu unterstützen und zu stärken.

Daher ging es in der Praxis darum:

- Eltern allgemein in der Erziehung zu unterstützen;

- Eltern dabei zu unterstützen, zu ihren Kindern sichere, verlässliche und empathische Beziehungen aufzubauen;
- Eltern und Kinder dabei zu unterstützen, Vertrauen in andere Menschen zu setzen;

- Eltern zu vermitteln, dass die Fähigkeit, sich an andere Menschen, aber auch an Werte zu binden, eine unterstützenswerte Kompetenz darstellt;

- Kinder in ihrer Identitätsentwicklung zu fördern;

- Eltern dabei zu unterstützen, ihre Werte bewusst zu reflektieren und Wertekonflikte aufzugreifen.

Wertevielfalt war und ist ein Kennzeichen des Projekts: Es wurden den Praxisstandorten keine inhaltlichen Vorgaben gemacht, welche Werte gefördert werden sollten. Dieser Ansatz gilt auch für die Umsetzungsstrategien: Es gab keine Vorgaben, mit welchen Strategien Wertebildung beziehungsweise Wertesensibilisierung unterstützt werden sollte.

Unter den 15 ausgewählten Standorten guter Praxis finden sich Familienbildungsstätten, Mehrgenerationenhäuser, Familienzentren und Eltern-Kind-Zentren, die zum Beispiel Kurse, Trainings, Gruppen, Treffs, Patenschaftsmodelle, Einzelaktivitäten oder Beratung anboten, so beispielsweise:

- Familienwochenenden

- Vorträge und Podiumsdiskussionen

- Broschüren, Flyer, Bücher

- Eltern- und Großelternkurse

- Eltern-Kind-Gruppen

- Väterarbeit

- Fortbildungen für Mitarbeiterinnen und Mitarbeiter

- Präsentationen in politischen Gremien

- Entwicklung spezieller Materialien

In der mit eineinhalb Jahren recht kurzen ersten Praxisphase fand auch eine enorme Vielzahl von Fachveranstaltungen Platz. Auch international wurde das Projekt bekannt gemacht, so zum Beispiel am 8. Juli 2009 auf dem Wirtschafts- und Sozialgipfel der Vereinten Nationen oder bei der Weltfamilienorganisation auf dem »World Family Summit WFS+5 « vom 4. bis 7. Dezember 2009 in Istanbul. 


\section{Evaluationsergebnisse}

Die begleitende Evaluation in der ersten Projektphase, durchgeführt von der Universität Erlangen-Nürnberg, brachte unter anderem folgende Ergebnisse:

- An 15 Projektstandorten wurden insgesamt 89 verschiedene Maßnahmen durchgeführt.

- Die Angebote erreichten in besonderem Maße Familien mit niedrigem sozialen Status, bildungsferne Familien sowie Wohngebiete mit besonderem Erneuerungsbedarf.

- 64 Prozent der Angebote erreichten Personen mit Migrationshintergrund.

- Die Teilnehmerinnen und Teilnehmer zeigten sich mit den Angeboten sehr zufrieden.

- Mehr als 90 Prozent der Einrichtungen gaben an, ihre Ziele »vollständig « oder "ziemlich « erreicht zu haben.

- Auch die Mitarbeiterinnen und Mitarbeiter an den Projektstandorten gaben an, dass das Projekt bei ihnen Sensibilisierungs- und Reflexionsprozesse bezogen auf ihre eigenen Werte ausgelöst habe.
Diese Ergebnisse machen deutlich, dass das Projekt die Menschen erreicht hat, und dass die Angebote insgesamt sehr gut angenommen und bewertet wurden.

\section{Projektphase 2: Juli 2010 bis Dezember 2011}

Unter dem Titel »Wertebildung in Familien: Förderung wertesensibilisierender pädagogischer Praxis in der Bildungsarbeit mit Familien « ging das in der ersten Phase aufgebaute Projektnetzwerk am 1. Juli 2010 in die zweite Phase. Kernziele der Projektphase 2 sind weitere Sensibilisierung für das Wertethema, Professionalisierung, Entwicklung neuer und Weiterentwicklung bewährter Angebote wertesensibilisierender Bildungsarbeit mit Familien, Sammlung, Aufbereitung und noch stärkere Bekanntmachung der Angebote, Qualifizierung von Multiplikatoren und Schulung von weiteren pädagogischen und sozialen Berufsgruppen sowie der Aufbau eines europäischen Netzwerks der Wertebildung. Thematisch fokussieren sich die Angebote des Projekts

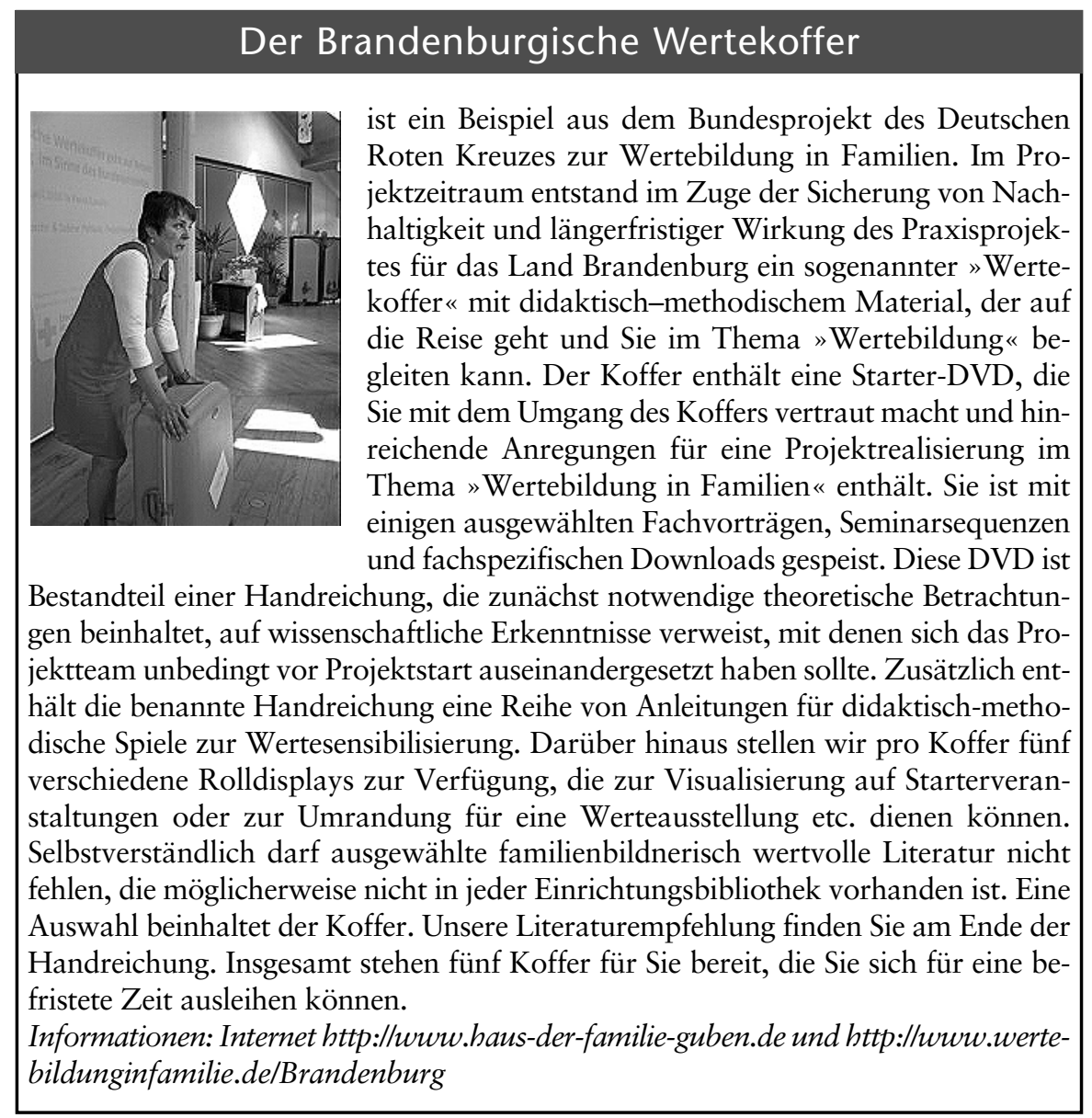

in der aktuellen Projektphase auf Gender und Interkulturalität.

Um diese ambitionierten Zielsetzungen zu erreichen, sind u. a. folgende Maßnahmen konzeptionell geplant oder bereits umgesetzt:

- Ausbau der Projektstandorte zu Kompetenzzentren

- Entwicklung und Durchführung wertesensibilisierender Fort- und Weiterbildungsangebote für pädagogische Berufsgruppen (z. B. Erzieherinnen und Erzieher; Lehrerinnen und Lehrer)

- Entwicklung und Durchführung von regionalen und landesweiten Fachveranstaltungen

- Gründung des Kompetenzforum Wertebildung (Standorte, Projektkoordination und Externe) als zentrales Projektgremium. Dieser regelmäßig tagende Kreis stellt projektintern die Schnittstelle zwischen Theorie und Praxis dar und soll gleichermaßen die Praktikerinnen und Praktiker themenorientiert mit neuem theoretischen Input versorgen, wie ihnen die Gelegenheit zu gemeinsamer intensiver Praxisreflexion bieten.

- Ausbau der Homepage, indem das Wertethema umfassend und aktuell präsentiert (Literaturlisten, Texte zum Download, Sammlung empirischer Ergebnisse u. a. m.) und der aktuelle Sachstand im Projekt regelmäßig dokumentiert werden soll.

- Sammlung und Aufbereitung der bisher im Projekt entwickelten Angebote und Veröffentlichung auf der Projekt-Homepage. So können die methodischen Bestände über die Grenzen des Projekts hinaus allen Interessierten zugänglich gemacht werden.

- Zum Ausbau des europäischen Netzwerks sind bereits mehrere Vorträge vor internationalem Publikum sowie der Ausbau internationaler Kooperationen in den Standorten geplant.

\section{Ausblick}

Bereits jetzt darf die Entwicklung in Projektphase 2, sowohl was das allgemeine Interesse als auch die Entwicklung der Praxis in den Projektstandorten angeht, als sehr positiv eingeschätzt werden. Laufend neue Informationen sind auf der Homepage erhältlich (Internet http:// www.wertebildunginfamilien.de). 